\title{
Rapid Active Zone Remodeling during Synaptic Plasticity
}

\author{
Annika Weyhersmüller, ${ }^{1 *}$ Stefan Hallermann, ${ }^{1 *}$ Nicole Wagner, ${ }^{2}$ and Jens Eilers ${ }^{1}$ \\ ${ }^{1}$ Carl Ludwig Institute of Physiology, Medical Faculty, University of Leipzig, 04103 Leipzig, Germany, and ${ }^{2}$ Institute of Physiology, University of Würzburg, \\ 97070 Würzburg, Germany
}

How can synapses change the amount of neurotransmitter released during synaptic plasticity? Although release in general is intensely investigated, its determinants during plasticity are still poorly understood. As a model for plastic strengthening of synaptic release, we here use the well-established presynaptic homeostatic compensation during interference with postsynaptic glutamate receptors at the Drosophila neuromuscular junction. Combining short-term plasticity analysis, cumulative EPSC analysis, fluctuation analysis, and quantal short-term plasticity modeling, we found an increase in the number of release-ready vesicles during presynaptic strengthening. High-resolution light microscopy revealed an increase in the amount of the active zone protein Bruchpilot and an enlargement of the presynaptic cytomatrix structure. Furthermore, these functional and structural alterations of the active zone were not only observed after lifelong but already after minutes of presynaptic strengthening. Our results demonstrate that presynaptic plasticity can induce active zone remodeling, which regulates the number of release-ready vesicles within minutes.

\section{Introduction}

Longstanding research aims at understanding the structural and functional changes that allow synapses to change their strength of transmission (Albright et al., 2000). The amount of neurotransmitter released is determined by two parameters (del Castillo and Katz, 1954; Murthy et al., 1997): the number of release-ready vesicles $(N)$ and the vesicular release probability $\left(p_{\mathrm{vr}}\right)$, the probability of each of them to be released upon an action potential. Synaptic release probability ( $p_{\text {syn }}$, i.e., the probability that at least one vesicle is released per whole synapse) is related to these two parameters by $p_{\text {syn }}=1-\left(1-p_{\mathrm{vr}}\right)^{N}$ (Hanse and Gustafsson, 2001b). The dissection of both parameters is essential for a coherent molecular understanding of alterations in neurotransmitter release during synaptic plasticity and has been addressed in a number of seminal studies. Early work on Aplysia, for example, shows alterations in active zone (AZ) size and number and in the number of associated vesicles during habituation or sensitization of the gill-withdrawal reflex (Bailey and Chen, 1983). In a pioneering study that combines electrophysiology with electron microscopy in the same neuron at the crayfish neuromuscular junction (NMJ), Wojtowicz et al. (1994) show that facilitated synapses have a larger number of AZs, accompanied by an increase in $N$. During long-term depression, the number of releaseready vesicles, estimated by hypertonic sucrose application, was found to be decreased (Goda and Stevens, 1998). Accordingly, an increase in synaptic release probability after several days of inac-

\footnotetext{
Received Dec. 21, 2010; revised Feb. 16, 2011; accepted Feb. 23, 2011.

Author contributions: A.W. and S.H. designed research; A.W., S.H., and N.W. performed research; A.W. and S.H. analyzed data; A.W., S.H., and J.E. wrote the paper.

${ }^{*}$ A.W. and S.H. contributed equally to this work.

We thank C. F. Stevens for helpful discussions, E. Hanse and R. J. Kittel for critically reading this manuscript, S. J. Sigrist for fly strains and the GluRIID antibody, and E. Buchner for the Nc82 antibody.

Correspondence should be addressed to Stefan Hallermann, Carl Ludwig Institute of Physiology, University of Leipzig, Liebigstrasse 27, 04103 Leipzig, Germany. E-mail: hallermann@medizin.uni-leipzig.de.

DOI:10.1523/JNEUROSCI.6698-10.2011

Copyright $\odot 2011$ the authors $\quad 0270-6474 / 11 / 316041-12 \$ 15.00 / 0$
}

tivity goes along with an increase in $N$ (Murthy et al., 2001). Finally, by combining FM dye loading with electron microscopy in hippocampal cell cultures, it has been demonstrated that synaptic release probability is correlated with the number of docked vesicles, which corresponds to the number of release-ready vesicles (Schikorski and Stevens, 2001; Branco et al., 2010).

Here we use the genetically, functionally, and structurally highly amenable glutamatergic NMJ of Drosophila melanogaster and focus on changes in neurotransmitter release induced by well-established forms of homeostatic plasticity, which offset perturbations in postsynaptic function (Turrigiano et al., 1998; Turrigiano, 2007; Pozo and Goda, 2010). Synaptic homeostasis at the Drosophila NMJ has become an extensively studied model system to analyze the molecular mechanisms of synaptic plasticity (Petersen et al., 1997; DiAntonio et al., 1999; Goda and Davis, 2003; Frank et al., 2006; Goold and Davis, 2007; Dickman and Davis, 2009; Frank et al., 2009; Bergquist et al., 2010). In this study, we aimed at resolving changes in $p_{\mathrm{vr}}$ and $N$ during synaptic plasticity at the Drosophila NMJ. We asked whether alterations in the structure and molecular composition of AZs could be resolved. We combine paired-pulse experiments, fluctuation analysis, high-frequency stimulation, and short-term plasticity modeling and show that the number of release-ready vesicles can increase within minutes. Furthermore, using confocal and stimulated emission depletion (STED) imaging, we find an increase in the amount of a core AZ protein, Bruchpilot (BRP) (Kittel et al., 2006; Wagh et al., 2006), as a morphological correlate for synaptic strengthening during both lifelong and minute timescale plastic changes.

\section{Materials and Methods}

Electrophysiology. We used white-1 flies and glutamate receptor IIA subunit (GluRIIA)/GluRIIB double mutants rescued by a GluRIIB transgene with its endogenous promoter (DiAntonio et al., 1999; Schmid et al., 2008). All flies were raised at $25^{\circ} \mathrm{C}$. Two-electrode voltage-clamp (TEVC) experiments were performed using an Axoclamp 2B amplifier (Molecular Devices) at room temperature $\left(22-24^{\circ} \mathrm{C}\right)$ on ventral longitudinal 
muscle 6 of male third-instar larvae in extracellular hemolymph-like solution HL3 (Stewart et al., 1994) containing the following (in mM): 70 $\mathrm{NaCl}, 5 \mathrm{KCl}, 10 \mathrm{NaHCO}_{3}, 5$ trehalose, 115 sucrose, 5 HEPES, and $1 \mathrm{CaCl}_{2}$ or (as indicated) $\mathrm{MgCl}_{2}$ adjusted to yield a total divalent ion concentration of $21 \mathrm{~mm}$. Recordings were made from cells with input resistances $\geq 4 \mathrm{M} \Omega$ and initial membrane potentials between -50 and $-70 \mathrm{mV}$. Intracellular electrodes with resistances of $10-35 \mathrm{M} \Omega$ filled with $3 \mathrm{M} \mathrm{KCl}$ were used. The holding potential was $-60 \mathrm{mV}$ for evoked EPSCs (eEP$\mathrm{SCs}$ ) and $-80 \mathrm{mV}$ for miniature EPSCs (mEPSCs). Nerve stimulation $(200 \mu \mathrm{s}, 1-10 \mathrm{~V})$ was performed with a Grass S 48 stimulator (Grass Technologies). The stimulation intensity was adjusted to reliably recruit both axons innervating muscle 6 and was comparable between the different groups (e.g., $7.6 \pm 1.6$ and $7.7 \pm 1.1 \mathrm{~V}, n=8$ and 6 , in controls and GluRIIB, respectively; $p=0.7$; in experiments of Fig. $1 D$ ). An additional increase of stimulation intensity did not increase the size of the eEPSC. Our eEPSC amplitudes were lower compared with previous studies (Kittel et al., 2006; Schmid et al., 2008; Hallermann et al., 2010a) because we stimulated the nerve at a more distant site to avoid electrotonic activation of $\mathrm{Ca}^{2+}$ channels (Ueda and $\mathrm{Wu}, 2009$ ). Consistent with previous findings (Ueda and $\mathrm{Wu}, 2009$ ), distant stimulation lead to significantly lower amplitudes compared with nerve stimulation closer to the NMJ in the same cell ( $44 \pm 5$ vs $71 \pm 9 \mathrm{nA}, n=4$ each; paired $t$ test, $p=0.03$ ). Single EPSCs were recorded at a stimulation frequency of $0.2 \mathrm{~Hz}$, and 10 EPSCs were averaged for each cell. The quantal content was determined as the ratio of eEPSC amplitudes to the mean mEPSC amplitudes, assuming that mEPSCs and eEPSCs arise from the same pool of vesicles. In a subset of experiments, quantal content was determined per cell (see Figs. $1 D$, $6 \mathrm{~B}$ ), leading to similar results. mEPSCs recorded at $-80 \mathrm{mV}$ holding potential were scaled to $-60 \mathrm{mV}$ assuming a reversal potential of $0 \mathrm{mV}$ and a linear current voltage relation. No TTX was used for mEPSC recordings, but mEPSCs could be distinguished from infrequently occurring spontaneous EPSCs by their size. For paired-pulse experiments, pulses were applied repetitively at interstimulus intervals of 10, 30, 100, 300 , and $1000 \mathrm{~ms}$, and 3-10 repetitions were averaged for each cell at each interstimulus interval. After subtracting the baseline mean within 10-90 $\mathrm{ms}$ before the onset of the EPSC, the current trace elicited by paired-pulse stimulation was fitted with the following function (Schmidt et al., 2003):

$I(t)$

$$
= \begin{cases}0 & t<d_{1} \\ k_{1}\left(1-\operatorname{Exp}\left[-\frac{t-d_{1}}{r_{1}}\right]\right) \operatorname{Exp}\left[-\frac{t-d_{1}}{f_{1}}\right] & d_{1} \leqslant t<d_{2} \\ k_{1}\left(1-\operatorname{Exp}\left[-\frac{t-d_{1}}{r_{1}}\right]\right) \operatorname{Exp}\left[-\frac{t-d_{1}}{f_{1}}\right] & d_{2} \leqslant t, \\ & +k_{2}\left(1-\operatorname{Exp}\left[-\frac{t-d_{2}}{r_{2}}\right]\right) \operatorname{Exp}\left[-\frac{t-d_{2}}{f_{2}}\right]\end{cases}
$$

where $d_{i}$ defines the delay and $r_{i}$ and $f_{i}$ the rise and decay time constants of the $i$ th EPSC $(i=1,2)$. To obtain the peak amplitude of the $i$ th EPSCs, the constants $k_{i}$ were defined as follows:

$$
k_{i}=a_{i}\left(f_{i}+r_{i}\right) f_{i}^{-1}\left(\frac{f_{i}+r_{i}}{r_{i}}\right)^{\frac{r_{i}}{f_{i}}} .
$$

With the constrain $r_{2}=r_{1}$ and $f_{2}=f_{1}$, the remaining six free parameters $a_{1}, a_{2}, d_{1}, d_{2}, r_{1}$, and $f_{1}$ were optimized with Igor Pro 6.1 (Wavemetrics), and the paired-pulse ratio (PPR) was defined as $a_{2} / a_{1}$. If the delays of any of the EPSCs (defined as $d_{i}$ subtracted by the time of the nerve stimulation) deviated more than $\pm 0.5 \mathrm{~ms}$ from the average delay within one experiment, the experiment was discarded. Data were recorded with pClamp10 (Molecular Devices) and analyzed with pClamp10, Igor Pro 6.1, and Mathematica 5 (Wolfram Research Ltd.).

For concanavalin A (conA) treatment, larvae were dissected and incubated for $30 \mathrm{~min}$ in HL3 containing $10 \mu \mathrm{M}$ conA type IV (Sigma). Afterward, recordings were made with conA $(10 \mu \mathrm{M})$ in the bath solution. The controls for these experiments were incubated in HL3 for $30 \mathrm{~min}$.
Fluctuation analysis. Approximately 20 EPSCs were elicited at different extracellular $\mathrm{Ca}^{2+}$ concentrations, ranging from 0.4 to $3 \mathrm{~mm}$. To determine the dependence of the mean EPSC amplitude on the extracellular $\mathrm{Ca}^{2+}$ concentration, the amplitude at each $\mathrm{Ca}^{2+}$ concentration was normalized to the EPSC amplitude at $1 \mathrm{mM} \mathrm{Ca}^{2+}$ in the same cell, and normalized amplitudes were then averaged across experiments (see Fig. $2 B$ ). The EPSC variance (and the variance of the variance) was calculated following Neher and coworkers (Meyer et al., 2001; Scheuss and Neher, 2001; Scheuss et al., 2002). The variance-mean plots for individual cells were fitted with $\operatorname{Var}(I)=I^{2} / N+q I$, where $I$ is the mean current amplitude, $q$ is the quantal size, and $N$ is the number of release-ready vesicles. $p_{\mathrm{vr}}$ at $1 \mathrm{mM} \mathrm{Ca}^{2+}$ was then calculated as $p_{\mathrm{vr}}=I /(N q)$. Intersite and intrasite quantal variability was estimated by the mean coefficient of variation $(\mathrm{CV})$ of mEPSCs and was similar in control and GluRIIB (see Fig. 2D). Because the effects of latency fluctuation (“jitter") nearly cancel those of quantal size variability (Taschenberger et al., 2005), correction for quantal variability is not necessary and was therefore not performed.

Analysis of miniature EPSCs. Low-noise recordings of mEPSCs were achieved by reducing electrode resistances to $<10 \mathrm{M} \Omega$ (otherwise 10-35 $\mathrm{M} \Omega$ ), lowering the amplifier gain, and increasing the phase lag. Spontaneous miniature EPSCs were identified by a template-detection method (Clements and Bekkers, 1997). Because fluctuation analysis yielded smaller values for quantal size than mEPSC measurements, we presumed that some of the smaller mEPSCs could not be distinguished from noise. To account for the mEPSCs that could not be resolved, we assumed a probability density function (pdf), $D(x)$, of the mEPSC amplitudes, $x$, proposed by Bekkers et al. (1990). The two parameters of the pdf [mean and variance (Bekkers et al. 1990)] were adjusted until both the mean of the $\operatorname{pdf}(\bar{X})$ was equal to the value of $q$ obtained in fluctuation analysis (see Fig. $3 E$, left) and the large-amplitude side of the measured histograms was reproduced by the pdf. $\bar{X}$ was calculated as $\bar{X}=\int_{0}^{\infty} x D(x) d x / \int_{0}^{\infty} D(x) d x$. Assuming equal CV of the mEPCS amplitudes, the pdf of GluRIIB mEPSCs was scaled down until $\bar{X}=q$ of GluRIIB animals. The predicted mean amplitude of detectable events (see Fig. 3G, left) was determined as $m=\int_{T}^{\infty} x D(x) d x / \int_{T}^{\infty} D(x) d x$, where $T$ is the detection threshold of $0.5 \mathrm{nA}$ (and $0.3 \mathrm{nA}$ in low noise recordings), based on the onset of the falling flank in the histogram of detected mEPSCs. To predict the measured frequency, we calculated the proportion of detected events (d) as $d=\int_{T}^{\infty} D(x) d x / \int_{0}^{\infty} D(x) d x$. The assumed frequency of mEPSCs (see Fig. 3E, right) in control was set to the measured frequency in controls divided by $d$. For simplicity, equal frequencies in GluRIIB and control animals were assumed. The predicted frequency of each group (Fig. $3 G$, right) was calculated as the product of the assumed frequency and $d$ of the corresponding group. Very similar values were obtained (data not shown) when distributions were calculated according to $\alpha$ functions or skewed Gaussian functions (Fraser and Suzuki, 1969; Rusch and Lelieur, 1973). Consistent with the obtained results from this approach, additional mEPSC recordings at a holding potential of $-100 \mathrm{mV}$ (i.e., with increased driving force and improved signal-to-noise ratio) yielded higher mEPSC frequencies and lower mEPSC amplitude estimates than recordings at $-80 \mathrm{mV}$ (when scaled to the reference potential $-60 \mathrm{mV}$ ).

To account for the overestimation in mEPSC amplitudes, we used the following correction relying on our results for $q$ from the fluctuation analysis. The measured mEPSC amplitude was multiplied with the ratio of $q$ and the mean of the detected mEPSCs, resulting in a correction factor of 0.65 and 0.46 for controls and GluRIIB animals, respectively.

The rate of false-positive events (i.e., noise peaks falsely classified as mEPSCs) for a simple threshold-detection method was estimated according to Equation 17 of Colquhoun and Sigworth (1995), taking into account the filtering characteristics of the cell $(400 \mathrm{~Hz}$, based on Colquhoun and Sigworth, 1995, their Eq. 10). The rate was 0.001 and $0.03 \mathrm{~Hz}$ for average and low noise measurements, respectively, well below the measured mEPSC frequency (see Fig. 3C). Furthermore, our rate of false-positive events is probably even lower, because the templatedetection method (Clements and Bekkers, 1997) takes other criteria into account, such as length and shape of events. 
Cumulative postsynaptic current analysis during high-frequency transmission. Short-term plasticity during and after high-frequency stimulations was investigated as described previously (Hallermann et al., 2010a): 100 pulses at $60 \mathrm{~Hz}$ were followed by test pulses at increasing intervals from $25 \mathrm{~ms}$ to $10 \mathrm{~s}$. The EPSC amplitudes in the train were measured from the peak to the baseline immediately before the onset of that EPSC. To calculate the quantal content in the train, we used mean mEPSC amplitudes measured before the train and scaled them by the correction factor obtained from fluctuation analysis (see above) in GluRIIB and controls [used for GluRIIB/philanthotoxin 433-treated animals (PhTx) and controls, respectively]. Note, if uncorrected mEPSCs were used for quantal content estimations, back-extrapolation yielded $440 \pm 40$ release-ready vesicles for GluRIIB animals and $240 \pm 30$ for controls $(n=$ 10 and 9, respectively; $p=0.002$ ) and $480 \pm 70$ and $240 \pm 70$ for PhTx and their controls, respectively ( $n=6$ and 5 , respectively; $p=0.03$ ) (insets in Figs. 2F, 6E).

Estimation of Bruchpilot amount. Third-instar larval preparations were stained with mouse Nc82 antibodies (1:200) against the presynaptic active zones protein Bruchpilot and rabbit antibodies to the postsynaptic glutamate receptor IID subunit (1:500), essentially as described previously (Schmid et al., 2008; Fouquet et al., 2009). Mutants and PhTxtreated animals were stained in the same well as their respective controls. Images were taken using a $60 \times$ water-immersion objective [numerical aperture (NA) 0.9; Olympus]. $Z$-stacks were made from the whole NMJ $6 / 7$ with oversampling in the $x-y$ plane (pixel distance, $120 \mathrm{~nm}$ ) and in $z$-direction $(\Delta z=200 \mathrm{~nm})$. The same laser intensity was used for all images of one genotype or treatment and the respective controls. Although we have no indications for differences in antibody permeability in mutants and controls, we cannot exclude this possible caveat.

To quantify the amount of BRP and GluRIID per synapse, we followed the procedures described previously (Schmid et al., 2008). In short, background-subtracted maximum projections of $Z$-stacks of both the Nc82 and the GluRIID image were scaled to a maximum of 255 arbitrary units (a.u.), and from the maximum projection of the two images, a binary mask was created by defining a threshold of 50 a.u. and converting all pixels below this threshold (i.e., outside the mask) to 0 . This procedure resulted in a mask of separated single synapses that was 0 anywhere but within the synaptic area. Confluent synapses in this mask were separated by hand. The fluorescence intensity in the (background-subtracted but unscaled) images was integrated for each individual synapse within this mask. Similarly, we quantified the mean BRP intensity of all AZs by using only the BRP image to create the mask and averaging the pixel intensity within the whole mask. In addition, the number and intensity of BRP peaks was analyzed using a peak-finding algorithm (finding local maxima after noise correction) in NIH ImageJ (W. S. Rasband, National Institutes of Health, Bethesda, MD; http://rsb.info.nih.gov/ij/, 1997-2009).

STED imaging. STED (Hell, 2007) images were taken with a $100 \times, 1.4$ NA oil objective (Leica) from NMJ 6/7 of control and GluRIIB thirdinstar larvae treated with mouse Nc82 antibodies (1:50) as described previously (Kittel et al., 2006; Fouquet et al., 2009). The Atto 647 dye attached to the secondary antibody was excited with a pulsed laser at 635 $\mathrm{nm}$ and depleted with a second laser adjusted to $760 \mathrm{~nm}$ (Mai Tai titanium:sapphire; Newport Spectra-Physics). The ring frequency was quantified by the ratio of the number of ring structures in each bouton to the total bouton area. The ring diameter was measured as the distance of the peaks in an intensity profile of a line through the center of the ring. The area of each $\mathrm{Nc} 82$ spot was determined by creating a mask as described above (threshold, 50 a.u. in images peak-scaled to 255 a.u.) and measuring the area of the spots. Image analysis was performed blind with respect to genotype.

Philanthotoxin treatment. The glutamate receptor blocker PhTx (Sigma) was prepared as a $4 \mathrm{~mm}$ stock solution in DMSO and diluted in HL3 to the final concentration of $10 \mu \mathrm{M}$ (final DMSO concentration, $0.25 \%$ ). To analyze the acute effects of PhTx, larval preparations were voltage clamped at $-60 \mathrm{mV}$, and $10 \mu \mathrm{M}$ PhTx was applied to the bath during the recording. For chronic PhTx treatment, semi-intact preparations were made as described previously (Frank et al., 2006) and incubated in $10 \mu \mathrm{M}$ PhTx for $10 \mathrm{~min}$. Controls were treated in the same way but were incubated in normal extracellular solution for $10 \min (n=27)$. After the $10 \mathrm{~min}$ incubation, the preparation was completed and TEVC recordings were made without PhTx in the bath solution.

Only preparations with mean mEPSC amplitudes at least $1 \mathrm{SD}$ below the mean mEPSC amplitude in controls were classified as undergoing sufficient postsynaptic blockade and were therefore included in the analysis, similar to the criterion applied by Dickman et al. (2009). Application of this criterion led to a rejection of 2 of 11 PhTx experiments. However, when all experiments were included in the analysis, the mEPSC amplitude was still significantly reduced $(0.70 \pm 0.03$ and $0.93 \pm 0.03 \mathrm{nA}, n=11$ and 27, in PhTx-treated animals and controls, respectively; $p<0.001$ ), and the quantal content was still significantly increased ( $74 \pm 9$ and $50 \pm 4, n=11$ and 27, in PhTx and controls, respectively; $p=0.02$ ). Note that the number of vesicles estimated by back-extrapolation of fits to the cumulative quantal content during high-frequency stimulation was also significantly increased in PhTx-treated compared with control animals without exclusion of any experiments $(1040 \pm 150$ and $370 \pm 110, n=6$ and 5, in PhTx and controls, respectively; $p=0.02$ ) (see Fig. $6 E$, inset).

Quantal short-term plasticity models. To estimate the number of release-ready vesicles and the vesicular release probability from shortterm plasticity during and after $60 \mathrm{~Hz}$ trains as well as from paired-pulse ratios recorded at different $\mathrm{Ca}^{2+}$ concentrations, we used constrained short-term plasticity models as described in detail previously (Hallermann et al., 2010a,b).

We first focused on a model with one pool of release-ready vesicles refilled from a finite supply pool [model 1 (see Fig. $8 \mathrm{~A}$ ), corresponding to Hallermann et al., 2010a, their model 6]. Model 1 is characterized by the following parameters: $\gamma$, the $\mathrm{Ca}^{2+}$ dependence of facilitation; $\alpha$, which defines the release probability; $N_{1}$, the number of readily releasable vesicles; $N_{0}$, the number of vesicles in the supply pool from which the readily releasable pool is refilled; and $k_{+1}, k_{-1}, k_{+0}$, and $k_{-0}$, the corresponding rates. In addition, we also used a model with two pools of release-ready vesicles and heterogeneous release probabilities (model 2) (see Fig. 8A) (Sakaba and Neher, 2001; Marrus and DiAntonio, 2004). In model 2, a small pool of release-ready vesicles $\left(N_{2}\right)$ with a high vesicular release probability $\left(p_{\mathrm{vr} 2}\right)$ is refilled from a larger pool $\left(N_{1}\right)$, which has a lower vesicular release probability $\left(p_{\mathrm{vr} 1}\right)$ and which, in turn, is refilled from a supply pool $\left(N_{0}\right)$. This model (corresponding to Hallermann et al., 2010a, their model 5a and Hallermann et al., 2010b, their model 3) reproduced the time course of short-term plasticity as well as the pairedpulse ratios at different $\mathrm{Ca}^{2+}$ concentrations (see Fig. $8 B-E$ ) better than model 1. In particular, the slow recovery of the PPR at $3 \mathrm{~mm}$ (which has not been measured in Hallermann et al., 2010a) was reproduced by this model but not by the simpler model 1 . There are two possible molecular mechanisms that could underlie model 2: first, two molecularly distinct pools of vesicles ("molecular" priming) or, second, two different distances of molecularly homogeneous vesicles to the nearest $\mathrm{Ca}^{2+}$ channels ("positional" priming) (Neher and Sakaba, 2008). In the latter scenario, a model with two pools represents most likely a simplification of a distribution of distances to the nearest $\mathrm{Ca}^{2+}$ channels and thus of a distribution of $p_{\mathrm{vr}}$.

Both models were first used to reproduce the average EPSC amplitudes during the $60 \mathrm{~Hz}$ train and recovery experiments as well as the PPRs for the different $\mathrm{Ca}^{2+}$ concentrations. For model 1 , only $N, p_{\mathrm{rv}}$, and $k_{+1}$, and, for model 2 , only $N_{1}, \alpha$ (defining $p_{\mathrm{vr} 1}$ and $p_{\mathrm{vr} 2}$ ), and $k_{+1}$ were used as free parameters. The remaining parameters were constrained to values estimated previously at the Drosophila NMJ (Hallermann et al., 2010a) with some adjustments because of the more distal stimulation of the axon (see above) (Ueda et al., 2009). In particular, the facilitation parameter $\gamma$ (Trommershäuser et al., 2003; Hallermann et al., 2010a) was set to 1 $\mu \mathrm{M}^{-1}$, to reproduce the paired-pulse facilitation at all investigated $\mathrm{Ca}^{2+}$ concentrations (see Fig. $8 D$, E). Subsequently, single experiments $(60 \mathrm{~Hz}$ train and recovery) were used to estimate the three free parameters of each model (Hallermann et al., 2010a). In each genotype and treatment, the best-fit parameters based on the average data including the PPRs (data not shown) were very similar to the average of the best-fit parameters based on the single experiments (see Fig. $8 F, G$ ). The third free parameter, $k_{+1}$, defining the rate of vesicle reloading per release site, was not significantly different between genotypes and treatment for both 
models (data not shown). Additionally constraining this parameter resulted in similar parameter estimates of $N$ and $p_{\text {rv }}$ (i.e., $N$ but not $p_{\text {rv }}$ was significantly increased for both models).

Statistical analysis. Analyses were performed with SigmaPlot 11 (Systat Software). The nonparametric Mann-Whitney rank sum test was used for statistical analysis unless stated otherwise. Asterisks indicate the significance level $\left.{ }^{*} p<0.05,{ }^{* *} p<0.01,{ }^{* * *} p<0.001\right)$. The data are reported as mean \pm SEM.

\section{Results}

Strong increase in quantal content but little change in paired-pulse ratio during presynaptic compensation Drosophila larvae lacking the GluRIIA and expressing only GluRIIB are a wellestablished genetic model system for homeostatic presynaptic strengthening (Petersen et al., 1997; DiAntonio et al., 1999; Frank et al., 2006; Dickman and Davis, 2009; Frank et al., 2009). In agreement with these studies, we found that the mutants showed strongly reduced amplitudes of spontaneous mEPSCs $(0.56 \pm 0.02$ and $0.93 \pm 0.08 \mathrm{nA}, n=8$ each in GluRIIB animals and controls, respectively; $p<$ 0.001 ) (Fig. $1 A, B$ ). In contrast, the amplitudes of eEPSCs were normal in GluRIIB during low-frequency $(0.2 \mathrm{~Hz})$ stimulation $(38 \pm 3$ and $42 \pm 3 \mathrm{nA}, n=13$ and 16 , in GluRIIB animals and controls, respectively; $p=0.5$ ) (Fig. $1 C, D)$. Assuming that quanta summate linearly in eEPCSs and that mEPSCs and eEPSCs arise from the same pool of vesicles (see below), the quantal content, i.e., the number of vesicles released per action potential, was estimated as the quotient of the eEPSC amplitude and the mEPSC amplitude. The quantal content was almost doubled in GluRIIB animals $(103 \pm 11$ and $63 \pm 8$ in GluRIIB animals and controls, $n=7$ and 6 , respectively; $p=$ 0.04) (Fig. 1D). In agreement with a previous study that showed stronger desensitization of GluRIIB (DiAntonio et al., 1999; Pawlu et al., 2004), we found a decrease in eEPSC decay time constants in GluRIIB animals (Fig. 1D). Because stronger desensitization might lead to an underestimation of the eEPSC amplitude and the quantal content in GluRIIB animals, we repeated the experiments in animals treated with conA, a blocker of desensitization of insect glutamate receptors (Mathers and Usherwood, 1976; Augustin et al., 2007). conA increased the eEPSC decay time constants (5.4 \pm 0.2 and $4.1 \pm 0.3 \mathrm{~ms}, n=8$ and 13 , in conA-treated animals and controls, respectively; $p=0.02$ ), confirming that it reduced desensitization of glutamate receptors. Again, we found a strong increase in quantal content in conA-treated GluRIIB animals compared with conA-treated controls $(101 \pm 11$ and $56 \pm 5, n=6$ and 8 , in conA-treated GluRIIB animals and conA-treated controls, respectively; $p=0.003)($ Fig. $1 F)$.

The elevation in quantal content, which was observed with and without pharmacological reduction of glutamate receptor
A

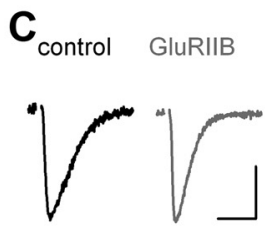

D

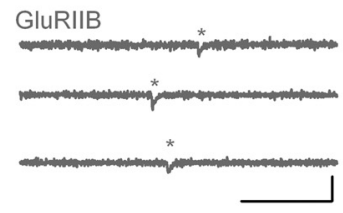

B

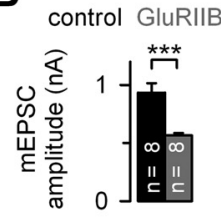

control GluRIIB
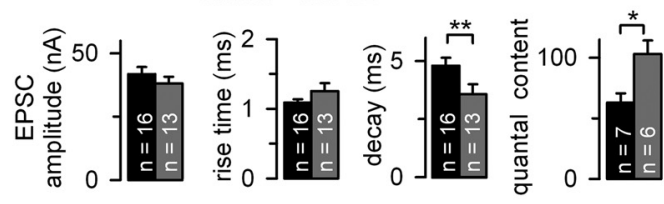

$\mathbf{F}$

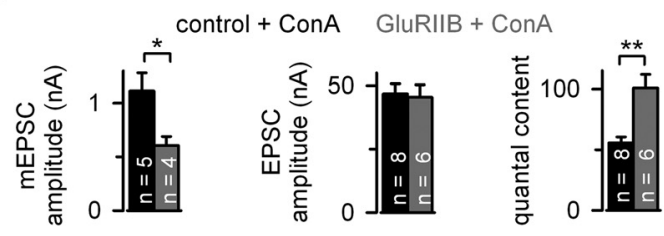

control GluRIIB
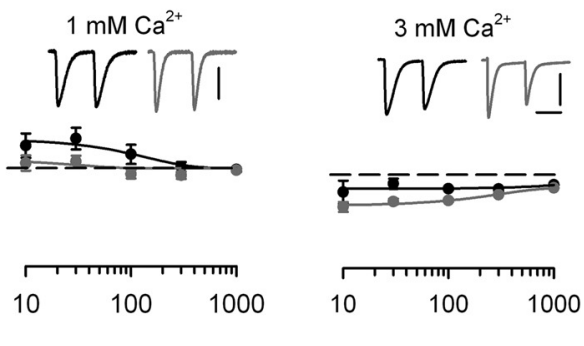

interstimulus interval (ms)

Figure 1. Strong increase in quantal content but little change in paired-pulse ratio during presynaptic compensation. $\boldsymbol{A}$,

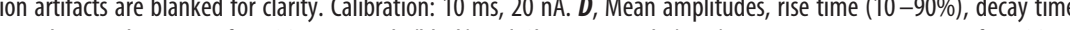
conA-treated $(10 \mu \mathrm{m})$ controls (black) and conA-treated GluRIIB animals (gray). Calibration: $10 \mathrm{~ms}, 20 \mathrm{nA}$. F, Mean mEPSC amplitude, eEPSC amplitude, and quantal content in conA-treated GluRIIB animals (gray) and conA-treated controls (black). G, PPR versus interstimulus interval at three different extracellular $\mathrm{Ca}^{2+}$ concentrations (as indicated) for control (black) and GluRIIIB animals (gray) superimposed with corresponding exponential fits (left, $n=5$ and 4; middle, $n=6$ and 8 ; right, $n=6$ and 6 , :5, 20, and $60 \mathrm{nA} \mathrm{from} \mathrm{left} \mathrm{to} \mathrm{right} \mathrm{and} 20 \mathrm{~ms}$. Inset (bottom), Illustration of the method for determining the amplitude (vertical gray lines) of the first and second EPSC for short interstimulus intervals ( 10 ms interstimulus); dashed horizontal line, baseline; solid gray line, exponential decay of the first EPSC; white line, fit to the measured EPSCs (see Eqs. 1, 2).

desensitization, could be attributable to an increase in either the number of release-ready vesicles or the release probability per vesicle. To discriminate between these two options, we analyzed PPRs, which critically depend on the initial vesicular release probability (Dobrunz and Stevens, 1997; Hanse and Gustafsson, 2001a), but also on the degree of facilitation (Katz and Miledi, 1968) as well as on receptor saturation and desensitization (for review, see Zucker and Regehr, 2002; Xu-Friedman and Regehr, 2004). Strikingly, PPRs at various interstimulus intervals showed little alterations in GluRIIB animals compared with controls in different extracellular $\mathrm{Ca}^{2+}$ concentrations (Fig. 1G). Although depression was significantly stronger in GluRIIB animals than in controls in $3 \mathrm{mM} \mathrm{Ca}^{2+}$ (three-way ANOVA with Holm-Sidak post hoc test, $p=0.02$ ) (Holm, 1979), the difference appeared small compared with the large increase in quantal content. Triggered by this finding, we further analyzed the mechanisms of 
A

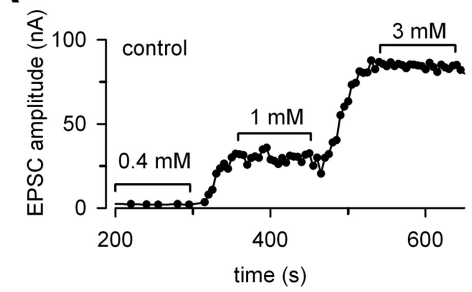

C

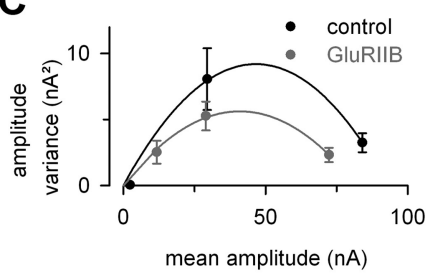

E

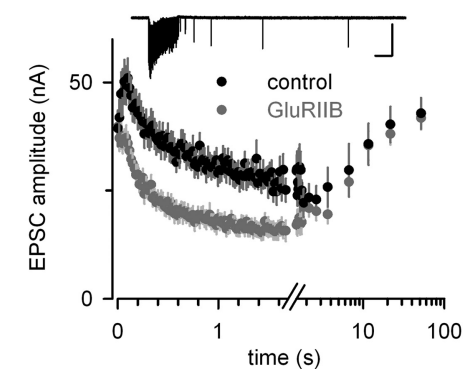

B

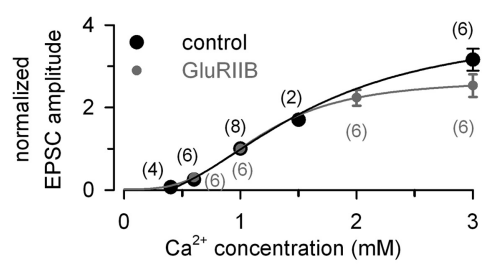

D

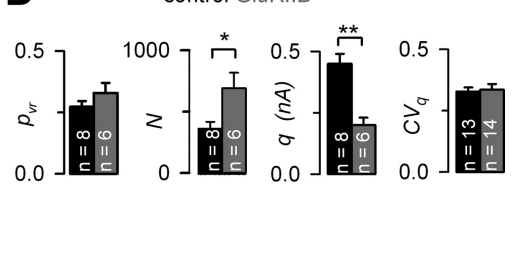

$\mathbf{F}$

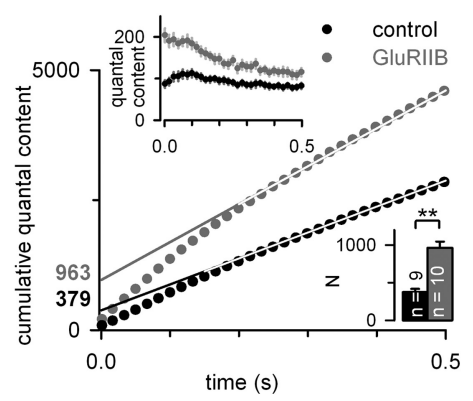

Figure 2. Increased number of release-ready vesicles during presynaptic strengthening. $\boldsymbol{A}$, Amplitudes of eEPSCs of a control example elicited at $0.2 \mathrm{~Hz}$ at the indicated extracellular $\mathrm{Ca}^{2+}$ concentrations. The brackets indicate regions used for subsequent analysis. $\boldsymbol{B}$, EPSC mean amplitudes normalized to the amplitude at $1 \mathrm{~mm} \mathrm{Ca}^{2+}$ plotted versus external $\mathrm{Ca}^{2+}$ concentration for control (black) and GluRIIB (gray) animals fitted with a Hill function according to $I_{\text {norm }}\left(\left[\mathrm{Ca}^{2+}\right]\right)=I_{\text {norm max }}\left[1+\left(\mathrm{EC}_{50} /\right.\right.$ $\left.\left.\left[\mathrm{Ca}^{2+}\right]\right)^{\text {slope }}\right]^{-1}$, which yielded $I_{\text {norm_max }}=3.9$ and $2.7, \mathrm{EC}_{50}=1.5$ and $1.2 \mathrm{~mm}$, and slope $=2.2$ and 3.1 for controls and GluRIIB animals, respectively. The number of experiments for each concentration is indicated. $C$, Examples of variance-mean plots with parabolic fits in control (black) and GluRIIB (gray). D, Mean quantal parameters vesicular release probability $\left(p_{\mathrm{vr}}\right)$ at $1 \mathrm{~mm}\left(\mathrm{a}^{2+}\right.$, number of release-ready vesicles $(N)$, and quantal size $(q)$ estimated from fluctuation analysis in control (black) and GluRIIB (gray); the mean coefficient of variation of $q\left(\mathrm{CV}_{\mathrm{q}}\right)$ was determined from mEPSC amplitudes. $\boldsymbol{E}$, Mean EPSC amplitudes during and at increasing intervals after high-frequency stimulation ( $60 \mathrm{~Hz}, 100$ pulses) for control (black; $n=10$ ) and GluRIIB (gray; $n=10$ ) animals. Note the logarithmic timescale after the train (broken abscissa). Inset, Example current trace of the first $15 \mathrm{~s}$ of a control experiment. Calibration: 1 s, 30 nA. Error bars indicate SEM. $\boldsymbol{F}$, Average cumulative quantal content in control (black; $n=9)$ and GluRIIB ( $g r a y ; n=10$ ) animals for the first $500 \mathrm{~ms}$ of the experiments shown in $\boldsymbol{E}$. Back-extrapolation of linear fits to the average cumulative quantal content at 300-500 ms (straight lines) yielded estimates for the readily releasable pool of 379 vesicles for control and 963 vesicles for GluRIIB animals. Top inset, Quantal content during the first $500 \mathrm{~ms}$ of the $60 \mathrm{~Hz}$ stimulus train in control (black) and GluRIIB (gray) animals. Bottom inset, Average release-ready vesicle estimates from back-extrapolation of the cumulative quantal content of single experiments in control (black) and GluRIIB (gray) animals ( $p=0.001$ ).

synaptic plasticity. In particular, we set out to examine whether mechanisms other than the previously described increase in vesicular release probability (Frank et al., 2006) could account for the increase in quantal content during presynaptic compensation.

\section{Increased number of release-ready vesicles during presynaptic strengthening}

We performed fluctuation analysis (Scheuss and Neher, 2001; Silver, 2003) to estimate the three quantal parameters: $N, p_{\mathrm{vr}}$, and the quantal size $q$. EPSCs were elicited at $\mathrm{Ca}^{2+}$ concentrations of $0.4-3 \mathrm{~mm}$ (Fig. $2 A, B$ ). $N, p_{\mathrm{vr}}$, and $q$ were extracted from parabolic fits to the variance-mean plot (see Materials and Methods) for each cell (Fig. 2C). Consistent with estimates from mEPSC measurements (Fig. 1, compare $A, B$ ), $q$ was reduced to $\sim 50 \%$ in GluRIIB animals $(0.20 \pm 0.03$ and $0.45 \pm 0.04 \mathrm{nA}$ in GluRIIB animals and controls, respectively; $p=0.001$ ) (Fig. $2 D$ ). Fluctuation analysis showed only an insignificant tendency toward an increase in $p_{\mathrm{vr}}(33 \pm 4$ and $27 \pm 2 \%, n=6$ and 8 , in GluRIIB animals and controls; $p=0.3$ ) (Fig. 2D), consistent with the slight difference in PPRs, whereas $N$ was almost doubled in GluRIIB animals (690 \pm 128 and $359 \pm 58$ in control; $p=0.03$ ) (Fig. $2 D)$. These results indicate that the increase in the number of release-ready vesicles contributes to the increased quantal content in GluRIIB animals.

As a different approach to estimate the number of release-ready vesicles, we used cumulative postsynaptic current analysis during high-frequency stimulus trains (100 pulses at $60 \mathrm{~Hz}$ ) in control and GluRIIB animals (Schneggenburger et al., 1999) (Fig. 2E,F). Back-extrapolation from linear fits to the cumulative quantal content of single experiments at 300-500 $\mathrm{ms}$ in the train (Fig. $2 F$ ) (Hallermann et al., 2010a) yielded $376 \pm 43$ and $964 \pm 82$ vesicles for control and GluRIIB animals, respectively ( $n=9$ and $10, p=0.001)$ (Fig. $2 F$, bottom inset) (for the calculation of the quantal content, see Materials and Methods and following section). This finding supports the hypothesis of an increased number of release-ready vesicles during presynaptic strengthening. The $N$ estimates from fluctuation analysis and back-extrapolation in controls are in accordance with electron microscopic investigations, in which the number of synapses onto muscle 6 in wild-type was estimated to $\sim 500$ (Atwood et al., 1993).

\section{Quantal size is overestimated by mEPSC recordings}

Because estimates of $q$ form fluctuation analysis were lower than the mean of measured mEPSCs, we assumed that smaller mEPSCs might have been missed because of limitations in the resolution. This conclusion is based on the assumption that mEPSCs and eEPSCs arise from the same pool of vesicles. In hippocampal cell cultures, this assumption has been questioned (Sara et al., 2005; Atasoy et al., 2008), but it appears unlikely that mEPSCs are approximately twice as large as the quantal events underlying eEPSCs (compare Figs. 1B, 2D). To test the hypothesis that smaller mEPSCs were not resolved, we performed additional mEPSC recordings in GluRIIB animals at reduced noise levels (achieved by altering pipette resistances and amplifier settings; see Materials and Methods). The recordings yielded lower mean amplitudes and higher frequencies of mEPSCs (Fig. 3A-C) compared with recordings at average noise levels, indicating that the real mEPSC distributions differed from the measured mEPSC distributions. To account for the differences, we assumed an mEPSC distribution as described by Bekkers et al. (1990) (Fig. 3D-F) (see Materials and Methods). The measured mEPSC amplitudes and frequencies could be well predicted by these calculated distributions (Fig. 3, compare $C, G$ ). To obtain correct quantal content estimates taking into account the overestimation in the quantal size, we used a correction factor 
obtained from our results in fluctuation analysis (see Analysis of miniature EPSCs in Materials and Methods). However, even without these corrections, all following conclusions were unaffected.

\section{Increased amount of Bruchpilot per synapse during presynaptic strengthening}

Next, we addressed the question whether the increase in release-ready vesicles goes along with morphological changes in the presynapse. To this end, we stained NMJs with Nc82 antibodies against the presynaptic protein BRP, a core component of the AZ (Kittel et al., 2006; Hallermann et al., 2010c). The number of BRP spots, counted as the number of peaks detected in the background-subtracted image (Fig. $4 B$, arrows), was not significantly different in GluRIIB and control animals (532 \pm 31 and $627 \pm 46, n=8$ each, in GluRIIB and control, respectively; $p=$ 0.1 ) (Fig. 4C). The intensity of the peaks, however, was increased in GluRIIB compared with control images $(157 \pm 8$ and $128 \pm 8$ a.u., $n=8$ each, in GluRIIB animals and controls, respectively; $p=0.02$ ) (Fig. $4 C$ ). The mean BRP pixel intensity across all AZs (Fig. 4B) (for exact procedure of quantification, see Materials and Methods) was also increased in GluRIIB animals (103 \pm 4 and $88 \pm 3$ a.u., $n=8$ each, in GluRIIB animals and controls; $p=0.01$ ) (Fig. 4C). These results show that the amount of BRP but not the number of AZs is increased at NMJs of GluRIIB animals.

To address the question whether the amount of BRP is increased at individual AZs apposed to the postsynaptic density of GluRIIB animals, we stained NMJs with Nc82 antibodies and simultaneously with antibodies to the postsynaptic GluRIID, an integral component of both IIA and IIB receptors (Qin et al., 2005). The amount of BRP and GluRIID per synapse was quantified by integrating the intensity over the synapse area (Schmid et al., 2008). The amount of BRP at individual synapses of GluRIIB animals was increased to $132 \%$ of control values ( $p<0.001$ ) (Fig. $4 D)$, whereas the number of postsynaptic receptors, determined as described previously (Schmid et al., 2008), was reduced (61 \pm 1 and $120 \pm 2, n=1868$ and 2452, in GluRIIB and control synapses, respectively; $p<0.001$ ) (Fig. $4 D$ ), probably attributable to the lack of IIA receptors without a compensatory increase in the number of IIB receptors. These data indicate that our electrophysiological finding of more release-ready vesicles is accompanied by an increased amount of the protein BRP per AZ at presynaptically strengthened synapses.

\section{STED analysis reveals increased BRP ring diameter during presynaptic strengthening}

The increased amount of BRP at individual synapses in GluRIIB animals is consistent with two different scenarios: first, it might reflect a larger number of BRP spots, which cannot be resolved as individual spots by confocal imaging because the distance between them is below the confocal optical resolution, or, second, the BRP spots might be bigger. To further address these possibilities, we took advantage of the high resolution of STED imaging (Hell, 2007). When imaged with deconvolution-enhanced STED microscopy, Nc82-labled BRP spots appear as ring-like structures at planar AZs (Fig. 5A) (Kittel et al., 2006; Fouquet et al., 2009; Hallermann et al., 2010c). There was a slight tendency toward an increase in ring frequency in GluRIIB compared with control (Fig. 5B). In contrast, both the area occupied by each BRP spot (Fig. $5 C$ ) and the diameter of the rings were significantly increased in GluRIIB animals (diameter, $152 \pm 5$ and $136 \pm 4 \mathrm{~nm}, n=93$ and 139, in GluRIIB and control, respectively; $p=0.005$ ) (Fig. $5 E$, determined as illustrated in $D$ and described in Materials and Methods). These data indicate that the increase in the number of release-ready vesicles is accompanied by an enlargement of individual BRP rings.

\section{Increased number of release-ready vesicles during rapid presynaptic strengthening}

The so far investigated mechanisms represent a lifelong adaptation to genetic interference with postsynaptic receptors, in which the time required to readjust the structure of the presynapse is not 
A
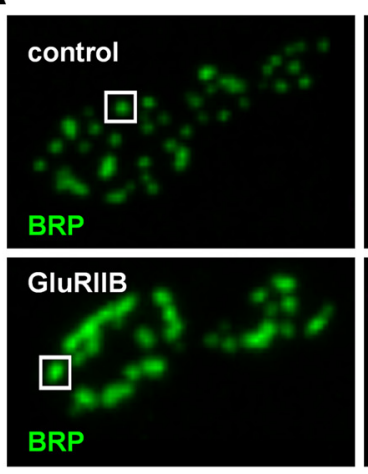

C

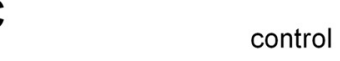

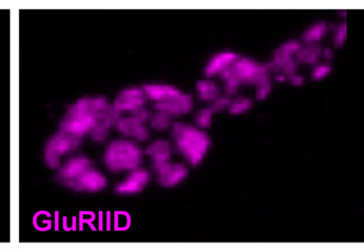

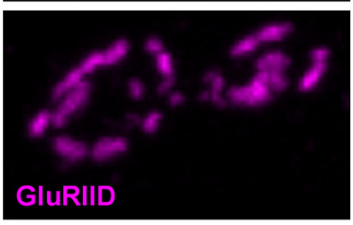

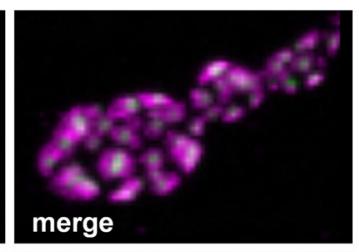

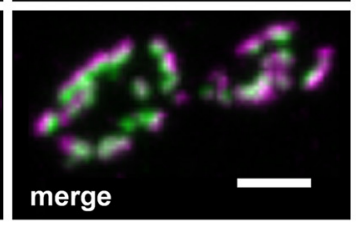

B
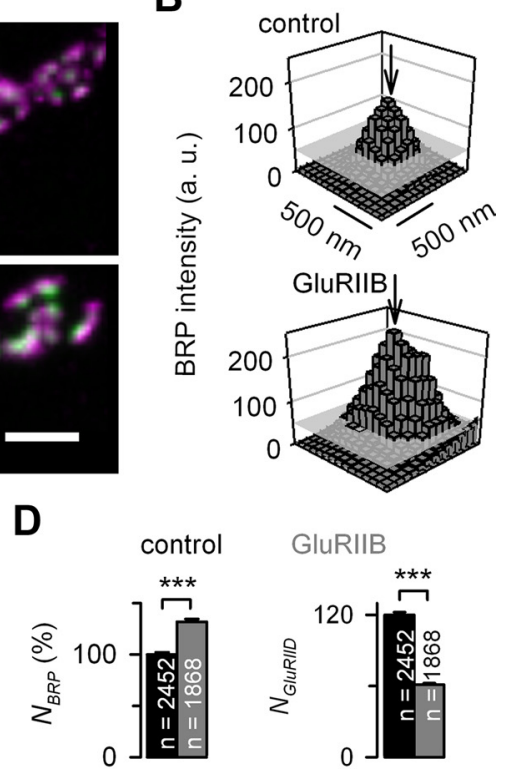

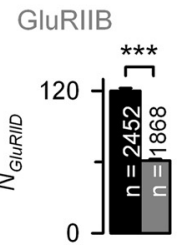

Figure 4. Increased amount of Bruchpilot during presynaptic strengthening. $A$, Examples of maximum projections of confocal Z-stacks of control (top) and GluRIIB (bottom) NMJs stained with Nc82 antibodies against the AZ protein BRP (green, left) and the postsynaptic GluRIID (magenta, middle) and merge of both (right). Scale bar, $5 \mu \mathrm{m}$. $\boldsymbol{B}$, BRP intensity in arbitrary units of the areas indicated by the white boxes in $A$ for control (top) and GluRIIB (bottom). The gray plane shows the threshold level ( 50 a.u.) used to isolate single BRP spots. Arrows indicate the estimated BRP peaks. C, Number of BRP peaks (left), mean intensity of the BRP peaks (middle), and mean BRP pixel intensity within BRP spots (right) in control (black) and GluRIIB animals (gray; $n=8 \mathrm{NMJs}$ from 8 different animals each). D, Quantification of BRP and GluRIIID amount at individual synapses in GluRIIB animals (gray; $n=1868$ synapses from 5 NMJs and 3 animals) and controls (black; $n=2452$ from $5 \mathrm{NMJs}$ and 3 animals), calculated by integrating the fluorescence intensity over the synapse area: BRP amounts are displayed in percentage of the average amount in control synapses; the number of postsynaptic receptors ( $N_{\text {GluRIII }}$ ) was estimated assuming an average of 120 receptors at control postsynapses (Schmid et al., 2008).

A
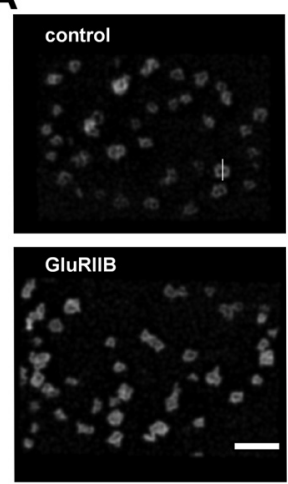
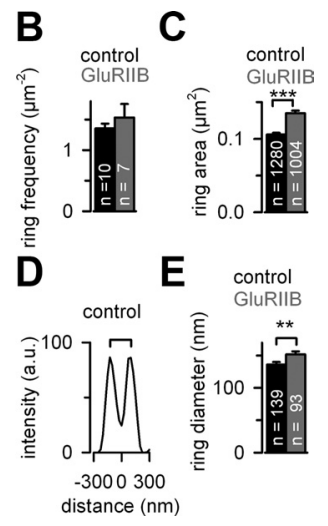

Figure 5. STED analysis reveals increased BRP ring diameter during presynaptic strengthening. $A$, STED images of a bouton of a control (top) and a GluRIIB animal (bottom) stained with $\mathrm{Nc} 82$ antibodies. Note the ring-shaped BRP structures. Scale bar, $1 \mu \mathrm{m}$. $\boldsymbol{B}$, Average ring frequency in GluRIIB animals (gray; $n=7$ NMJs from 5 animals) and controls (black; $n=10$ NMJs from 5 animals). C, The area of the BRP rings is increased in GluRIIB animals (gray; $n=1004$ rings and from $7 \mathrm{NMJs}$ and 5 animals) compared with control animals (black; $n=1280$ rings from $10 \mathrm{NMJs}$ and 5 animals). $\boldsymbol{D}$, Intensity profile of the line in $\boldsymbol{A}$. The ring diameter was quantified as the distance of the intensity peaks of a line profile through the center of the ring as illustrated by the brackets. $\boldsymbol{E}$, The average ring diameter is increased in GluRIIB (gray; $n=93$ rings from $5 \mathrm{NMJs}$ and 5 animals) compared with control (black; $n=139$ rings from $5 \mathrm{NMJs}$ and 5 animals).

the limiting factor. However, quantal content has been shown previously to increase within minutes after blockade of postsynaptic receptors by PhTx (Frank et al., 2006; Dickman and Davis, 2009). We therefore investigated whether the mechanisms that underlie lifelong synaptic strengthening also control rapid forms of plasticity.

To verify the blockade of postsynaptic glutamate receptors, we measured eEPSCs and mEPSCs for several minutes before and dur-

ing bath application of $10 \mu \mathrm{M}$ PhTx. The amplitude of both eEPSCs and mEPSCs was rapidly (with a time constant of $16 \mathrm{~s}$ ) and strongly reduced after wash in of PhTx compared with baseline measurements (Fig. 6A,B). To induce presynaptic compensation, we applied $10 \mu \mathrm{M}$ PhTx to semi-intact larval preparations as described (Frank et al., 2006); for controls, normal extracellular solution was applied. After 10 min of incubation, the preparation was completed and measurements were performed in the absence of PhTx (Fig. 6A, right). Although $\mathrm{PhTx}$ was not present in the bath during the recordings, mEPSCs were still significantly smaller in PhTx-treated animals compared with controls $(0.67 \pm 0.02$ and $0.93 \pm 0.03 \mathrm{nA}, n=9$ and 27, in PhTx and controls, respectively; $p<0.001$ ) (Fig. 6B), confirming previous findings of an irreversible component of the postsynaptic receptor block (Frank et al., 2006). However, evoked EPSCs were not reduced in PhTx-treated animals ( $37 \pm 4$ and $34 \pm 3 \mathrm{nA}, n=9$ and 27, in PhTx and controls, respectively; $p=0.6$ ) (Fig. $6 B$ ), indicating an increase in quantal content $(86 \pm 10$ and $50 \pm 4$, in PhTx and controls, respectively; $p=0.03$ ) (Fig. 6B). To analyze the mechanisms of this rapid form of synaptic strengthening, short-term plasticity was analyzed in the same way as described for GluRIIB animals. The results in PhTxtreated larvae were strikingly similar to those in GluRIIB animals (Fig. 6C-E). Paired-pulse ratios were almost identical in PhTxtreated and control animals (compare Figs. 6C, 1G). Readilyreleasable pool estimates from back-extrapolation were $370 \pm 110$ vesicles for control and $1050 \pm 180$ vesicles for PhTx-treated animals $(n=5$ each; $p=0.03$ ) (compare Figs. $6 E$, bottom inset, $2 F)$. When experiments were performed with PhTx remaining in the bath, the results were similar (data not shown), except that EPSC and mEPSC amplitudes were smaller as a result of the reversible component of the PhTx block (Frank et al., 2006). These results thus indicate that compensation is achieved by changes in the number of release-ready vesicles even on the timescale of minutes. 
Increased amount of BRP during rapid presynaptic strengthening

To examine whether 10 min presynaptic strengthening also goes along with an increase in the amount of BRP at the presynapse, we stained NMJs of PhTx-treated animals and their controls with $\mathrm{Nc} 82$ antibodies (Fig. 7A). The intensity of BRP peaks and the mean BRP pixel intensity was significantly increased at NMJs of PhTx-treated animals (peak intensity, $118 \pm 10$ and $90 \pm 5$ a.u., $n=8$ and 10, in PhTx-treated and control animals, respectively, $p<0.05$; mean pixel intensity, $95 \pm 4$ and $85 \pm 2$ a.u., $n=8$ and 10, in PhTx and controls, $p=0.02)($ Fig. $7 B)$. These findings indicate that lifelong and rapid presynaptic strengthening are associated with an increase in the amount of BRP.

\section{Quantal short-term plasticity models confirm increased number of release- ready vesicles during presynaptic strengthening}

Our results from fluctuation analysis and cumulative postsynaptic current analysis showed a strong increase in $N$ during plastic strengthening of release, whereas $p_{\mathrm{vr}}$ was only slightly increased. We therefore wondered whether the alterations in short-term plasticity during presynaptic strengthening (Figs. 1G, 2E, 6C,D) could be explained by the increase in $N$. To estimate $N$ and the $p_{\mathrm{vr}}$ from the short-term plasticity during high-frequency stimulation and from the paired-pulse experiments, we aimed to reproduce the experimental data with constrained short-term plasticity models (Fig. 8) (Hallermann et al., 2010a,b). First, we used a model with one pool of release-ready vesicles, which is refilled from a finite reserve pool (model 1) (Fig. 8A) (see Material and Methods). The estimates of $N$ from this model were significantly increased in GluRIIB and PhTx-treated animals compared with their respective controls (Fig. 8F, G). Vesicular release probability was not significantly increased (Fig. $8 F, G$ ), consistent with our data from fluctuation analysis. This model, however, did not predict the slow recovery of PPR in $3 \mathrm{mM}$ $\mathrm{Ca}^{2+}$ at longer interstimulus intervals (Fig. 8D,E). We therefore used a second model with two pools of release-ready vesicles $\left(N_{1}\right.$ and $\mathrm{N}_{2}$ ) with different vesicular release probabilities (model 2) (Fig. 8A) (Sakaba and Neher, 2001; Marrus and DiAntonio, 2004). The total number of release-ready vesicles (i.e., $N_{1}+N_{2}$ ) estimated by this model increased from $370 \pm 70$ in controls to $680 \pm 90$ in GluRIIB (Fig. 8F) and from $410 \pm 95$ in PhTx treatment controls to $1010 \pm 170$ in PhTx-treated animals (Fig. $8 G$ ), consistent with fluctuation analysis and cumulative postsynaptic current analysis (Figs. 2, 6). Thus, both short-term plasticity models further confirm the increase in the number of releaseready vesicles during both lifelong and minute timescale presynaptic plasticity.
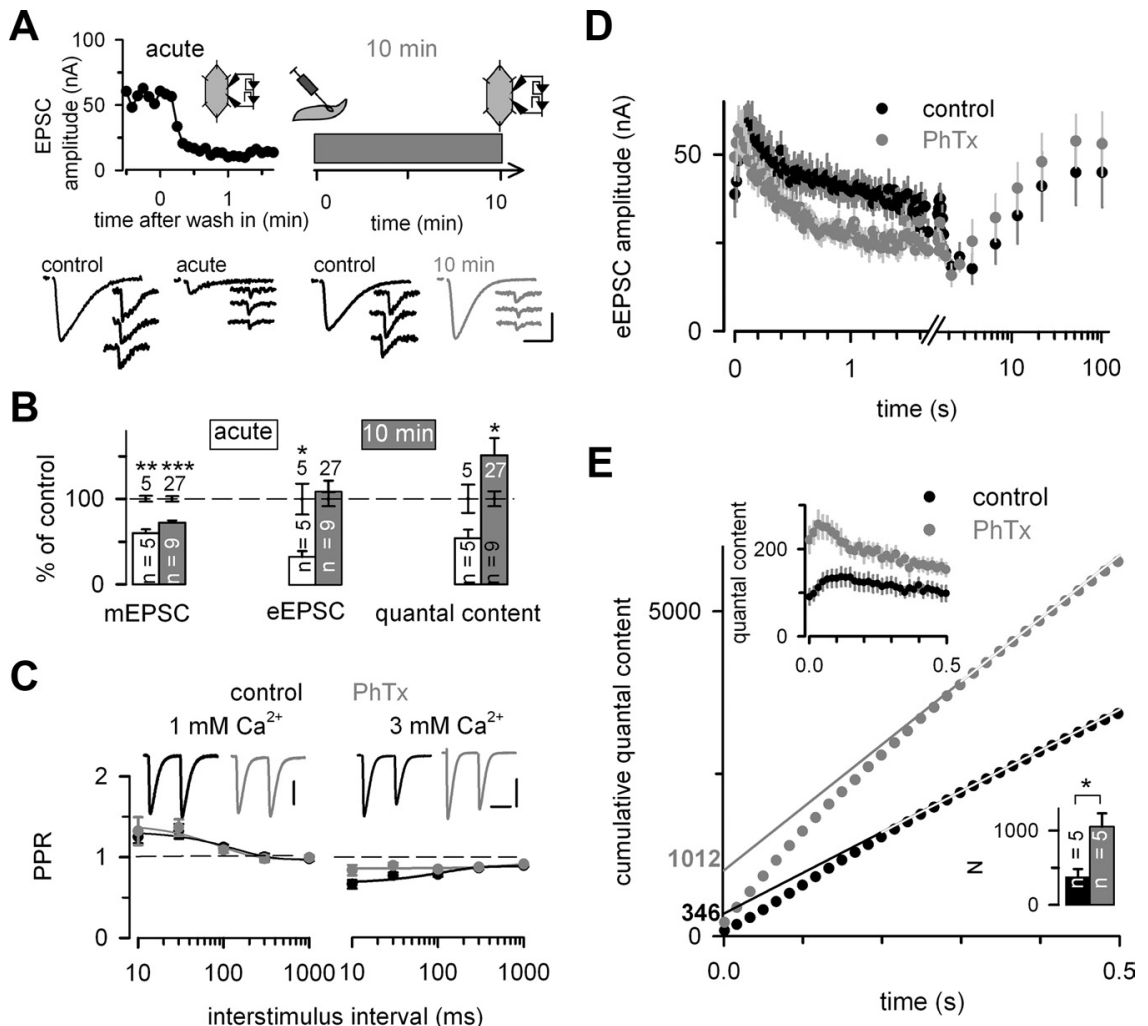

Figure 6. Increased number of release-ready vesicles during rapid presynaptic strengthening. $\boldsymbol{A}$, Illustration of acute $\mathrm{PhTx}$ application and $10 \mathrm{~min}$ PhTx pretreatment experiments. Left (acute), Example of an experiment in which PhTx was bath applied during TEVC recordings at $t=0$. The inset illustrates the recording condition in a larval preparation. eEPSC and mEPSC example traces before and $1 \mathrm{~min}$ after bath applich shown below. Right (10 min), Semi-intact preparations of larvae were incubated for 10 min in either normal extracellular solution or $10 \mu \mathrm{mPhTx}$. Afterward, the preparation was completed and mEPSCand eEPSC amplitudes were measured without Pin the extracellular solution. The insets illustrate the PhTx application to the semi-intact larva and the TEVC recording in the complete 列

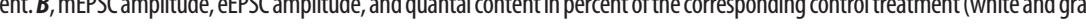
for acute and $10 \mathrm{~min}$ PhTx-treatment, respectively). The number of animals of the corresponding controls are indicated. C, Mean pairedpulse ratios versus interstimulus intervals fitted with a monoexponential function at $1 \mathrm{~mm}$ extracellular $\mathrm{Ca}^{2+}$ (left; $n=14$ and $\left.n=7\right)$ and (right; $n=6$ and $n=4$ ) for control (black) and PhTx (gray), respectively. Inset, Example traces at 30 ms interstimulus interval. Calibration: left, $20 \mathrm{nA}$; right: $60 \mathrm{nA}, 20 \mathrm{~ms}$. D, eEPSC amplitudes during and after high-frequency stimulation (100 pulses at $60 \mathrm{~Hz}$ ) in control (black; $n=5$ ) and PhTx-treated (gray; $n=5$ ) animals. Note the logarithmic timescale after the train (broken abscissa). $\boldsymbol{E}_{i}$

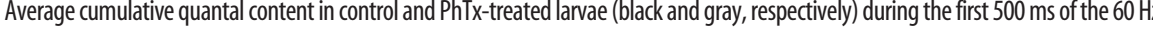
train shown in $\boldsymbol{D}$; back-extrapolation of linear fits to the average cumulative quantal content at $300-500 \mathrm{~ms}$ (straight lines) yielded 列 346 and 1012 release-ready vesicles for control and PhTx, respectively. Top inset, Quantal content during the first $500 \mathrm{~ms}$ in the $60 \mathrm{~Hz}$ stimulus train in control (black) and PhTx-treated (gray) animals. Bottom inset, Average release-ready vesicle estimates from backextrapolation of the cumulative quantal content of single experiments in control (black) and PhTx-treated (gray) animals ( $p=0.03)$.

\section{Discussion}

We have investigated the mechanisms that allow presynaptic terminals to change their amount of neurotransmitter release during synaptic plasticity. Fluctuation analysis, cumulative postsynaptic current analysis, and quantal short-term plasticity modeling revealed that the number of release-ready vesicles was almost doubled in synapses that compensated for postsynaptic deficits. Furthermore, the amount of the AZ protein BRP was increased. Thus, we provide evidence that the amount of neurotransmitter released can be increased by a dynamic network of AZ proteins, which rapidly recruits more functional release-ready vesicles to the AZ (Fig. $8 H$ ).

Vesicular release probability versus number of release-ready vesicles during plasticity

Our finding of an increase in the number of release-ready vesicles $(N)$ during plastic presynaptic strengthening is consistent with 
A
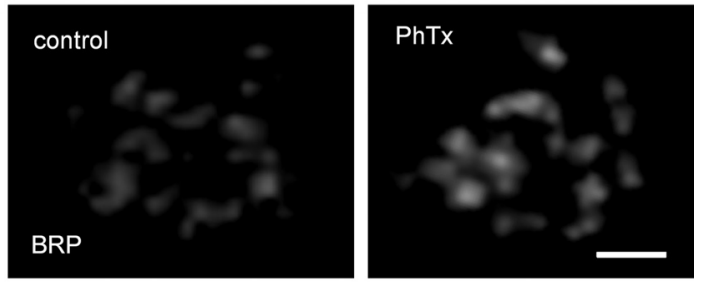

B

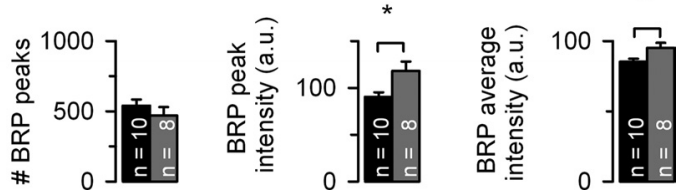

Figure 7. Increased amount of BRP during rapid presynaptic strengthening. $\boldsymbol{A}$, Examples of maximum projections of confocal Z-stacks of control (left) and PhTx (right) NMJs stained with $\mathrm{Nc} 82$ antibodies against BRP. Scale bar, $1 \mu \mathrm{m}$. B, Number of BRP peaks (left), mean intensity of the BRP peaks (middle), and mean BRP pixel intensity within BRP spots (right) in control (black; $n=10$ NMJs from 5 animals) and PhTx-treated (gray; $n=8$ NMJs from 4 animals) animals.

studies in hippocampal cell cultures, in which synaptic release probability $\left(p_{\text {syn }}\right)$ correlates with $N$ (Goda and Stevens, 1998; Murthy et al., 2001; Branco et al., 2010). It also corresponds well to the finding that prolonged depolarization leads to a reduction of both $N$ and $p_{\mathrm{vr}}$ (Moulder et al., 2004). Moreover, in a recent study, Matz et al. (2010) used genetically expressed pHluorin to examine $p_{\text {syn }}$ in hippocampal cell cultures. They find that $p_{\text {syn }}$ changes spontaneously on a timescale of minutes. Using fluorescence labeling of the AZ protein Bassoon, they also show that the size of the AZ cytomatrix is correlated with $p_{\text {syn }}$. Our study extends theses findings from spontaneous fluctuations of the state of the synapse to changes precisely compensating for reduced postsynaptic neurotransmitter sensitivity during synaptic plasticity.

Our functionally determined number of release-ready vesicle at the junction onto muscle $6[\sim 400$ (Fig. 2)] is similar to the number of AZs in serial electron microscopic images of the junction onto this muscle [ 500 (Atwood et al., 1993)]. Furthermore, these numbers are consistent with our confocal imaging [ $\sim 600$ AZs onto muscles 6 and 7, i.e., $\sim 300$ onto each muscle (Fig. 4)], taking into account that some AZs were not resolved with confocal imaging. Furthermore, extrapolations from AZs counted per bouton with STED microscopy (Fig. 5) revealed similar numbers ( $\sim 500 \mathrm{AZs})$. These data indicate that each AZ harbors approximately one release-ready vesicle under resting conditions. Interestingly, a recent study at the NMJ of the mouse found approximately two release-ready vesicles per AZ with similar techniques (Ruiz et al., 2011). In our experiments in which presynaptic strengthening was induced, the number of AZs estimated with confocal and STED imaging remained constant, but the number of functionally determined release-ready vesicles doubled, indicating that a presynaptically strengthened Drosophila AZ harbors approximately two release-ready vesicles (Fig. $8 H$ ).

Our quantal analyses of the GluRIIB phenotype and the PhTx treatment might represent an overestimation of the increase in $p_{\mathrm{vr}}$ (and a corresponding underestimation of the increase in $N$ ) for the following reason: postsynaptic receptor desensitization is more rapid in GluRIIB animals (DiAntonio et al., 1999; Pawlu et al., 2004) (Fig. 1D), and PhTx application probably also increases the impact of desensitization [see more rapid decay of mEPSCs and eEPSCs (Fig. 6A) and previous reports of a preference of PhTx for GluRIIA receptors (Frank et al., 2006)]. This accelerated desensitization in GluRIIB and PhTx-treated animals may lead to more depression during repetitive stimulation (Frank et al., 2006 and this study) and, at high extracellular $\mathrm{Ca}^{2+}$ in the fluctuation analyses, to lower EPSC amplitudes. In addition, as a result of the increased amount of neurotransmitter released in GluRIIB and PhTx-treated animals, the probability of glutamate receptor saturation is increased. Both effects will lead to more depression during repetitive stimulation and thus to an overestimation of $p_{\mathrm{rv}}$ in GluRIIB and PhTx-treated animals.

Detailed studies investigating presynaptic mechanisms of homeostasis compensation show an involvement of $\mathrm{Ca}^{2+}$ channels (Frank et al., 2006, 2009). This is consistent with our finding of an increase in the number of release-ready vesicles, because more vesicles with normal $p_{\mathrm{vr}}$ can be expected to require more $\mathrm{Ca}^{2+}$ channels per AZ for docking within microdomains and nanodomains of $\mathrm{Ca}^{2+}$ channels. Interestingly, the recruitment of more BRP molecules might be causally related to the observed involvement of $\mathrm{Ca}^{2+}$ channels (Frank et al., 2006, 2009), considering the proposed ability of BRP molecules to cluster $\mathrm{Ca}^{2+}$ channels (Kittel et al., 2006; Fouquet et al., 2009).

\section{Does homeostatic plasticity at the Drosophila NMJ activate silent synapses?}

Under resting conditions, some synaptic specializations identifiable by structure (e.g., close membrane apposition and postsynaptic density) do not exhibit functional transmission and are thus referred to as silent (for review, see Malenka and Nicoll, 1997; Atwood and Wojtowicz, 1999). It has been shown that these silent synapses can be turned into active ones by plastic changes, such as long-term potentiation (LTP) (Isaac et al., 1995; Liao et al., 1995). This mechanism has also been suggested for the crayfish NMJ, in which long-term facilitation leads to an increase in the binomial parameter $N$ (Wojtowicz and Atwood, 1986; Wojtowicz et al., 1994). It would therefore be possible that synaptic plasticity at the Drosophila NMJ activates either presynaptically silent ("mute") or postsynaptically silent ("deaf") synaptic contacts. Although synaptic unsilencing during LTP is mostly considered as a postsynaptic phenomenon (Isaac et al., 1995; Liao et al., 1995; Durand et al., 1996), there is also evidence for presynaptic components contributing to at least some forms of LTP (Kullmann et al., 1996; Tong et al., 1996; Reid et al., 2004; Sola et al., 2004). A recent study demonstrates that many release sites were silent during low-frequency stimulation (Peled and Isacoff, 2010). Furthermore, silent synapses can be rapidly reactivated by phosphorylation of synapsin (Doussau et al., 2010). A similar mechanism could be proposed for rapid synaptic plasticity. Because both presynaptic and postsynaptic unsilencing would lead to an increase in the number of synaptic contacts contributing to neurotransmitter release without changing quantal size or vesicular release probability, our electrophysiological finding of an increase in the number of release-ready vesicles would be in accordance with this interpretation. However, postsynaptic unsilencing would involve the recruitment of additional glutamate receptors to the postsynaptic density. In contrast, we found an $\sim 50 \%$ reduction in the number of glutamate receptors in GluRIIB animals, which corresponded well to the $\sim 50 \%$ reduction in quantal size. We therefore consider it unlikely that synaptic compensation is attributable to postsynaptic unsilencing. Presynaptic unsilencing, conversely, would be consistent with our electrophysiological findings. We therefore cannot exclude that the activation of presynaptically silent synaptic contacts contributes to synaptic plasticity, but our STED data favor the interpretation that individual preexisting and functional synaptic 
contacts are strengthened because they show an increase in the amount of BRP at each AZ [see also increased ring diameter (Fig. 5E)].

\section{The speed of cytomatrix reorganization} at the $\mathrm{AZ}$

The electron-dense cytomatrix at the AZ, also termed "T-bar" in Drosophila because of its T-shaped appearance in electron microscopic images, is assumed to be an important plasticity module (Wojtowicz et al., 1994; Cooper et al., 1996; Sigrist et al., 2003; Wichmann and Sigrist, 2010). We found that the amount of BRP, an integral component of the T-bar (Kittel et al., 2006; Fouquet et al., 2009), can be increased within minutes at NMJs. It might seem surprising that BRP, a large structural protein, can be recruited to AZs at this timescale. For example, it has been reported that the fluorescently labeled large vertebrate cytomatrix protein Bassoon recovers slowly after photobleaching $(>8 \mathrm{~h}$ ) in cultured hippocampal neurons (Tsuriel et al., 2009), suggesting that Bassoon constitutes a relatively stable presynaptic core scaffold and that BRP would show similar turnover rates. However, rapid structural remodeling of synapses has been demonstrated previously. Another recent study at cultured hippocampal neurons found that the amount of fluorescently labeled Bassoon molecules at AZs changes on a timescale of minutes (Matz et al., 2010), suggesting a very dynamic structure of the cytomatrix. Furthermore, at postsynaptic densities, structural remodeling on the timescale of even milliseconds has been shown to influence paired-pulse ratios by diffusional exchange of desensitized and un-desensitized glutamate receptors (Heine et al., 2008). A recent study at the Drosophila NMJ demonstrates that the late expression of the small GTPase Rab3 recruits large amounts of BRP molecules to Rab3-mutant AZs within hours (Graf et al., 2009). These data indicate that the cytomatrix at the AZ is indeed remarkably plastic, in particular when taking into account that the rate-limiting step might have been the expression of Rab3. Thus, our hypothesis that BRP amount can be precisely and rapidly adjusted at AZs within minutes is consistent with recent studies at both vertebrate and invertebrate AZs.

Based on our findings and previously published structural data (Koenig and Ikeda, 1999; Fouquet et al., 2009), we propose a model (Fig. 8 H) in which BRP molecules can be rapidly recruited to $\mathrm{AZs}$, leading to a larger number of releaseready vesicles as well as $\mathrm{Ca}^{2+}$ channels, consistent with the finding that BRP clusters $\mathrm{Ca}^{2+}$ channels (Kittel et al., 2006; Fouquet et al., 2009) as well as vesicles (Hallermann et al., 2010c). In summary, our data indicate that presynaptic strengthening is ac-
B

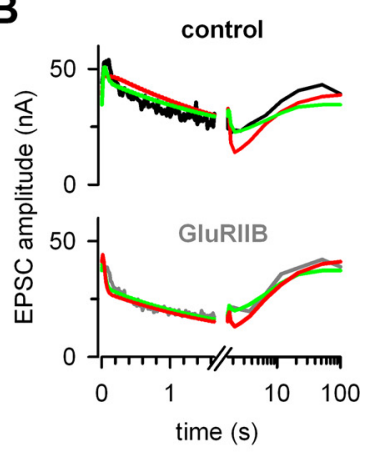

C

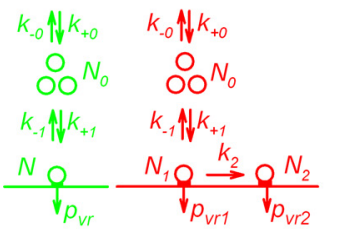

model $1 \quad$ model 2

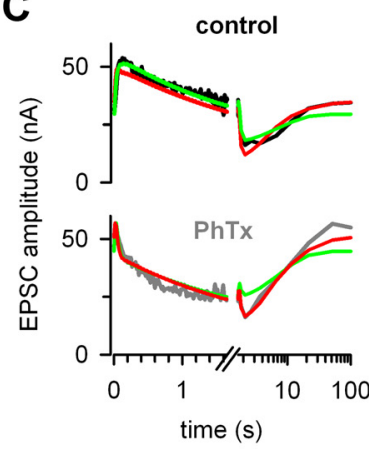

E control

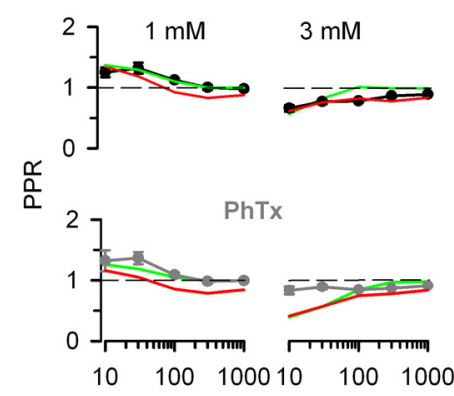

interstimulus interval (ms)

G

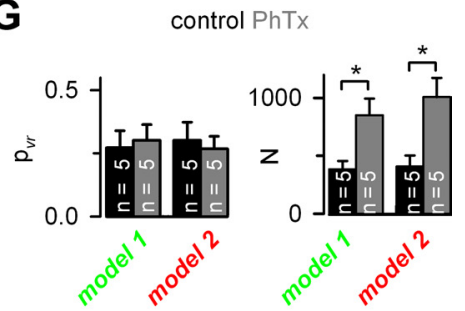

GluRIIB or PhTx
H

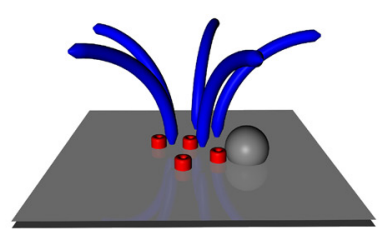

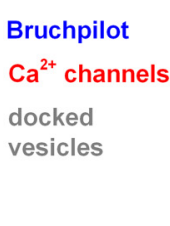

Figure 8. Quantal short-term plasticity models confirm increased number of release-ready vesicles during presynaptic strengthening. A, Illustration of short-term plasticity models. B, Average EPSC amplitudes during and after the $60 \mathrm{~Hz}$ train in control (black) and GluRIIB (gray) animals superimposed with the predictions from model 1 (green) and model 2 (red). C, Corresponding illustration of model predictions for PhTx-treated animals and their controls. D, Average paired-pulse ratios at three different $\mathrm{Ca}^{2+}$ concentrations for control and GluRIIB animals superimposed with the model predictions (color codes as in $\boldsymbol{B}$ ). $\boldsymbol{E}$, Corresponding paired-pulse experiments in the indicated $\mathrm{Ca}^{2+}$ concentrations in PhTx-treated animals and their controls superimposed on the model predictions (color codes as in $\boldsymbol{C}$ ). $\boldsymbol{F}$, Estimates of the parameters $p_{\mathrm{vr}}$ and $N$ obtained by fitting single $60 \mathrm{~Hz}$ experiments with model 1 (left) and model 2 (right) for control (black) and GluRIIB (gray) animals. For model $2, N$ refers to $N_{1}+N_{2}$ and $p_{\mathrm{vr}}$ to the average release probability $\left(p_{\mathrm{vr} 1} N_{1}+p_{\mathrm{vr} 2} N_{2}\right) /\left(N_{1}+N_{2}\right) . \mathbf{G}$, The same data as in $\boldsymbol{F}$ for PhTx-treated animals (gray) and controls (black). $\boldsymbol{H}$, Illustration of the hypothesized mechanism of presynaptic compensation. During lifelong and rapid presynaptic strengthening in GluRIIB and PhTx-treated animals, respectively, additional BRP molecules are recruited to the $\mathrm{AZ}$, leading to a larger cytomatrix, more $\mathrm{Ca}^{2+}$ channels, and more release-ready vesicles.

complished by an increase in the number of release-ready vesicles and structural remodeling at the $\mathrm{AZ}$ on a timescale of minutes.

\section{References}

Albright TD, Jessell TM, Kandel ER, Posner MI (2000) Neural science: a century of progress and the mysteries that remain. Neuron [Suppl] 25:S1-S55. 
Atasoy D, Ertunc M, Moulder KL, Blackwell J, Chung C, Su J, Kavalali ET (2008) Spontaneous and evoked glutamate release activates two populations of NMDA receptors with limited overlap. J Neurosci 28:10151-10166.

Atwood HL, Wojtowicz JM (1999) Silent synapses in neural plasticity: current evidence. Learn Mem 6:542-571.

Atwood HL, Govind CK, Wu CF (1993) Differential ultrastructure of synaptic terminals on ventral longitudinal abdominal muscles in Drosophila larvae. J Neurobiol 24:1008-1024.

Augustin H, Grosjean Y, Chen K, Sheng Q, Featherstone DE (2007) Nonvesicular release of glutamate by glial XCT transporters suppresses glutamate receptor clustering in vivo. J Neurosci 27:111-123.

Bailey CH, Chen M (1983) Morphological basis of long-term habituation and sensitization in Aplysia. Science 220:91-93.

Bekkers JM, Richerson GB, Stevens CF (1990) Origin of variability in quantal size in cultured hippocampal neurons and hippocampal slices. Proc Natl Acad Sci U S A 87:5359-5362.

Bergquist S, Dickman DK, Davis GW (2010) A hierarchy of cell intrinsic and target-derived homeostatic signaling. Neuron 66:220-234.

Branco T, Marra V, Staras K (2010) Examining size-strength relationships at hippocampal synapses using an ultrastructural measurement of synaptic release probability. J Struct Biol 172:203-210.

Clements JD, Bekkers JM (1997) Detection of spontaneous synaptic events with an optimally scaled template. Biophys J 73:220-229.

Colquhoun D, Sigworth FJ (1995) Fitting and statistical analysis of singlechannel records. In: Single-channel recording, Ed 2 (Sakmann B, Neher E, eds), pp 483-589. New York: Plenum.

Cooper RL, Winslow JL, Govind CK, Atwood HL (1996) Synaptic structural complexity as a factor enhancing probability of calcium-mediated transmitter release. J Neurophysiol 75:2451-2466.

del Castillo J, Katz B (1954) Quantal components of the end-plate potential. J Physiol 124:560-573.

DiAntonio A, Petersen SA, Heckmann M, Goodman CS (1999) Glutamate receptor expression regulates quantal size and quantal content at the Drosophila neuromuscular junction. J Neurosci 19:3023-3032.

Dickman DK, Davis GW (2009) The schizophrenia susceptibility gene dysbindin controls synaptic homeostasis. Science 326:1127-1130.

Dobrunz LE, Stevens CF (1997) Heterogeneity of release probability, facilitation, and depletion at central synapses. Neuron 18:995-1008.

Doussau F, Humeau Y, Benfenati F, Poulain B (2010) A novel form of presynaptic plasticity based on the fast reactivation of release sites switched off during low-frequency depression. J Neurosci 30:16679-16691.

Durand GM, Kovalchuk Y, Konnerth A (1996) Long-term potentiation and functional synapse induction in developing hippocampus. Nature 381:71-75.

Fouquet W, Owald D, Wichmann C, Mertel S, Depner H, Dyba M, Hallermann S, Kittel RJ, Eimer S, Sigrist SJ (2009) Maturation of active zone assembly by Drosophila Bruchpilot. J Cell Biol 186:129-145.

Frank CA, Kennedy MJ, Goold CP, Marek KW, Davis GW (2006) Mechanisms underlying the rapid induction and sustained expression of synaptic homeostasis. Neuron 52:663-677.

Frank CA, Pielage J, Davis GW (2009) A presynaptic homeostatic signaling system composed of the Eph receptor, Ephexin, $\mathrm{Cdc42}$, and $\mathrm{Ca}_{\mathrm{V} 2.1} \mathrm{cal}-$ cium channels. Neuron 61:556-569.

Fraser RDB, Suzuki E (1969) Resolution of overlapping bands. Functions for simulating band shapes. Anal Chem 41:37-39.

Goda Y, Davis GW (2003) Mechanisms of synapse assembly and disassembly. Neuron 40:243-264.

Goda Y, Stevens CF (1998) Readily releasable pool size changes associated with long term depression. Proc Natl Acad Sci U S A 95:1283-1288.

Goold CP, Davis GW (2007) The BMP ligand Gbb gates the expression of synaptic homeostasis independent of synaptic growth control. Neuron 56:109-123.

Graf ER, Daniels RW, Burgess RW, Schwarz TL, DiAntonio A (2009) Rab3 dynamically controls protein composition at active zones. Neuron 64:663-677.

Hallermann S, Heckmann M, Kittel RJ (2010a) Mechanisms of short-term plasticity at neuromuscular active zones of Drosophila. HFSP J 4:72-84.

Hallermann S, Fejtova A, Schmidt H, Weyhersmüller A, Silver RA, Gundelfinger ED, Eilers J (2010b) Bassoon speeds vesicle reloading at a central excitatory synapse. Neuron 68:710-723.

Hallermann S, Kittel RJ, Wichmann C, Weyhersmüller A, Fouquet W, Mertel S,
Owald D, Eimer S, Depner H, Schwärzel M, Sigrist SJ, Heckmann M (2010c) Naked dense bodies provoke depression. J Neurosci 30:14340-14345.

Hanse E, Gustafsson B (2001a) Paired-pulse plasticity at the single release site level: an experimental and computational study. J Neurosci 21:8362-8369.

Hanse E, Gustafsson B (2001b) Vesicle release probability and pre-primed pool at glutamatergic synapses in area CA1 of the rat neonatal hippocampus. J Physiol 531:481-493.

Heine M, Groc L, Frischknecht R, Béique JC, Lounis B, Rumbaugh G, Huganir RL, Cognet L, Choquet D (2008) Surface mobility of postsynaptic AMPARs tunes synaptic transmission. Science 320:201-205.

Hell SW (2007) Far-field optical nanoscopy. Science 316:1153-1158.

Holm S (1979) A simple sequentially rejective multiple test procedure. Scand J Stat 6:65-70.

Isaac JT, Nicoll RA, Malenka RC (1995) Evidence for silent synapses: implications for the expression of LTP. Neuron 15:427-434.

Katz B, Miledi R (1968) The role of calcium in neuromuscular facilitation. J Physiol 195:481-492.

Kittel RJ, Wichmann C, Rasse TM, Fouquet W, Schmidt M, Schmid A, Wagh DA, Pawlu C, Kellner RR, Willig KI, Hell SW, Buchner E, Heckmann M, Sigrist SJ (2006) Bruchpilot promotes active zone assembly, $\mathrm{Ca}^{2+}$ channel clustering, and vesicle release. Science 312:1051-1054.

Koenig JH, Ikeda K (1999) Contribution of active zone subpopulation of vesicles to evoked and spontaneous release. J Neurophysiol 81:1495-1505.

Kullmann DM, Erdemli G, Asztély F (1996) LTP of AMPA and NMDA receptor-mediated signals: evidence for presynaptic expression and extrasynaptic glutamate spill-over. Neuron 17:461-474.

Liao D, Hessler NA, Malinow R (1995) Activation of postsynaptically silent synapses during pairing-induced LTP in CA1 region of hippocampal slice. Nature 375:400-404.

Malenka RC, Nicoll RA (1997) Silent synapses speak up. Neuron 19:473-476.

Marrus SB, DiAntonio A (2004) Preferential localization of glutamate receptors opposite sites of high presynaptic release. Curr Biol 14:924-931.

Mathers DA, Usherwood PN (1976) Concanavalin A blocks desensitisation of glutamate receptors on insect muscle fibres. Nature 259:409-411.

Matz J, Gilyan A, Kolar A, McCarvill T, Krueger SR (2010) Rapid structural alterations of the active zone lead to sustained changes in neurotransmitter release. Proc Natl Acad Sci U S A 107:8836-8841.

Meyer AC, Neher E, Schneggenburger R (2001) Estimation of quantal size and number of functional active zones at the calyx of Held synapse by nonstationary EPSC variance analysis. J Neurosci 21:7889-7900.

Moulder KL, Meeks JP, Shute AA, Hamilton CK, de Erausquin G, Mennerick S (2004) Plastic elimination of functional glutamate release sites by depolarization. Neuron 42:423-435.

Murthy VN, Sejnowski TJ, Stevens CF (1997) Heterogeneous release properties of visualized individual hippocampal synapses. Neuron 18:599-612.

Murthy VN, Schikorski T, Stevens CF, Zhu Y (2001) Inactivity produces increases in neurotransmitter release and synapse size. Neuron 32:673-682.

Neher E, Sakaba T (2008) Multiple roles of calcium ions in the regulation of neurotransmitter release. Neuron 59:861-872.

Pawlu C, DiAntonio A, Heckmann M (2004) Postfusional control of quantal current shape. Neuron 42:607-618.

Peled ES, Isacoff EY (2011) Optical quantal analysis of synaptic transmission in wild-type and rab3-mutant Drosophila motor axons. Nat Neurosci 14:519-526.

Petersen SA, Fetter RD, Noordermeer JN, Goodman CS, DiAntonio A (1997) Genetic analysis of glutamate receptors in Drosophila reveals a retrograde signal regulating presynaptic transmitter release. Neuron 19:1237-1248.

Pozo K, Goda Y (2010) Unraveling mechanisms of homeostatic synaptic plasticity. Neuron 66:337-351.

Qin G, Schwarz T, Kittel RJ, Schmid A, Rasse TM, Kappei D, Ponimaskin E, Heckmann M, Sigrist SJ (2005) Four different subunits are essential for expressing the synaptic glutamate receptor at neuromuscular junctions of Drosophila. J Neurosci 25:3209-3218.

Reid CA, Dixon DB, Takahashi M, Bliss TV, Fine A (2004) Optical quantal analysis indicates that long-term potentiation at single hippocampal mossy fiber synapses is expressed through increased release probability, 
recruitment of new release sites, and activation of silent synapses. J Neurosci 24:3618-3626.

Ruiz R, Cano R, Casañas JJ, Gaffield MA, Betz WJ, Tabares L (2011) Active zones and the readily releasable pool of synaptic vesicles at the neuromuscular junction of the mouse. J Neurosci 31:2000-2008.

Rusch PF, Lelieur JP (1973) Analytical moments of skewed Gaussian distribution functions. Anal Chem 45:1541-1543.

Sakaba T, Neher E (2001) Quantitative relationship between transmitter release and calcium current at the calyx of Held synapse. J Neurosci 21:462-476.

Sara Y, Virmani T, Deák F, Liu X, Kavalali ET (2005) An isolated pool of vesicles recycles at rest and drives spontaneous neurotransmission. Neuron 45:563-573.

Scheuss V, Neher E (2001) Estimating synaptic parameters from mean, variance, and covariance in trains of synaptic responses. Biophys J 81:1970-1989.

Scheuss V, Schneggenburger R, Neher E (2002) Separation of presynaptic and postsynaptic contributions to depression by covariance analysis of successive EPSCs at the calyx of Held synapse. J Neurosci 22:728-739.

Schikorski T, Stevens CF (2001) Morphological correlates of functionally defined synaptic vesicle populations. Nat Neurosci 4:391-395.

Schmid A, Hallermann S, Kittel RJ, Khorramshahi O, Frölich AM, Quentin C, Rasse TM, Mertel S, Heckmann M, Sigrist SJ (2008) Activity-dependent site-specific changes of glutamate receptor composition in vivo. Nat Neurosci 11:659-666.

Schmidt H, Stiefel KM, Racay P, Schwaller B, Eilers J (2003) Mutational analysis of dendritic $\mathrm{Ca}^{2+}$ kinetics in rodent Purkinje cells: role of parvalbumin and calbindin $\mathrm{D}_{28 \mathrm{k}}$. J Physiol 551:13-32.

Schneggenburger R, Meyer AC, Neher E (1999) Released fraction and total size of a pool of immediately available transmitter quanta at a calyx synapse. Neuron 23:399-409.

Sigrist SJ, Reiff DF, Thiel PR, Steinert JR, Schuster CM (2003) Experiencedependent strengthening of Drosophila neuromuscular junctions. J Neurosci 23:6546-6556.

Silver RA (2003) Estimation of nonuniform quantal parameters with multiple-probability fluctuation analysis: theory, application and limitations. J Neurosci Methods 130:127-141.

Sola E, Prestori F, Rossi P, Taglietti V, D’Angelo E (2004) Increased neurotransmitter release during long-term potentiation at mossy fibregranule cell synapses in rat cerebellum. J Physiol 557:843-861.

Stewart BA, Atwood HL, Renger JJ, Wang J, Wu CF (1994) Improved sta- bility of Drosophila larval neuromuscular preparations in haemolymphlike physiological solutions. J Comp Physiol A Neuroethol Sens Neural Behav Physiol 175:179-191.

Taschenberger H, Scheuss V, Neher E (2005) Release kinetics, quantal parameters and their modulation during short-term depression at a developing synapse in the rat CNS. J Physiol 568:513-537.

Tong G, Malenka RC, Nicoll RA (1996) Long-term potentiation in cultures of single hippocampal granule cells: a presynaptic form of plasticity. Neuron 16:1147-1157.

Trommershäuser J, Schneggenburger R, Zippelius A, Neher E (2003) Heterogeneous presynaptic release probabilities: functional relevance for short-term plasticity. Biophys J 84:1563-1579.

Tsuriel S, Fisher A, Wittenmayer N, Dresbach T, Garner CC, Ziv NE (2009) Exchange and redistribution dynamics of the cytoskeleton of the active zone molecule bassoon. J Neurosci 29:351-358.

Turrigiano G (2007) Homeostatic signaling: the positive side of negative feedback. Curr Opin Neurobiol 17:318-324.

Turrigiano GG, Leslie KR, Desai NS, Rutherford LC, Nelson SB (1998) Activity-dependent scaling of quantal amplitude in neocortical neurons. Nature 391:892-896.

Ueda A, Wu CF (2009) Role of rut adenylyl cyclase in the ensemble regulation of presynaptic terminal excitability: reduced synaptic strength and precision in a Drosophila memory mutant. J Neurogenet 23:185-199.

Wagh DA, Rasse TM, Asan E, Hofbauer A, Schwenkert I, Dürrbeck H, Buchner S, Dabauvalle MC, Schmidt M, Qin G, Wichmann C, Kittel R, Sigrist SJ, Buchner E (2006) Bruchpilot, a protein with homology to ELKS/ CAST, is required for structural integrity and function of synaptic active zones in Drosophila. Neuron 49:833-844.

Wichmann C, Sigrist SJ (2010) The active zone T-bar: a plasticity module? J Neurogenet 24:133-145.

Wojtowicz JM, Atwood HL (1986) Long-term facilitation alters transmitter releasing properties at the crayfish neuromuscular junction. J Neurophysiol 55:484-498.

Wojtowicz JM, Marin L, Atwood HL (1994) Activity-induced changes in synaptic release sites at the crayfish neuromuscular junction. J Neurosci 14:3688-3703.

Xu-Friedman MA, Regehr WG (2004) Structural contributions to shortterm synaptic plasticity. Physiol Rev 84:69-85.

Zucker RS, Regehr WG (2002) Short-term synaptic plasticity. Annu Rev Physiol 64:355-405. 